Article

\title{
Group-Birefringence-Dispersion Measurements for Polarization-Maintaining Fibers Using a Kerr Phase-Interrogator
}

\author{
Yang Lu \\ College of Meteorology and Oceanography, National University of Defense Technology, Changsha 410073, \\ China; luyang01@nudt.edu.cn
}

Received: 24 November 2020; Accepted: 14 December 2020; Published: 17 December 2020

\begin{abstract}
A method which utilizes a Kerr phase-interrogator to measure the group birefringence dispersion (GBD) of a polarization-maintaining fiber (PMF) is systematically studied in this paper. The differential group delay of two sinusoidally modulated optical signals (SMOSs) polarized along the principal axes of the PMF is measured by a Kerr phase-interrogator, which leads to the group birefringence of the PMF. As the laser wavelength of the SMOSs varies, the group birefringence as a function of the laser wavelength is obtained, and the GBD is calculated as the derivative of the group birefringence with respect to the laser wavelength. The proposed method is experimentally demonstrated by characterizations of a Panda PMF with high GBD and an elliptical core PMF with low GBD, and its performance is analyzed. The proposed method eliminates the impact of the laser coherent length and allows for characterizing the GBD of PMFs that are tens of kilometers long.
\end{abstract}

Keywords: polarization-maintaining fiber; group birefringence dispersion; Kerr effect

\section{Introduction}

Polarization-maintaining fibers (PMFs) with lengths of hundreds of meters have been applied in many fields, such as ultrafast all-optical switching and ultrashort pulse generation [1,2]. In these applications, the group birefringence dispersion (GBD) of a PMF governs the temporal profiles of amplitudes and spectra of optical pulses through nonlinear optical effects. The characterization of the GBD of the PMF helps to control the properties of the optical pulses. PMFs have also been employed in distributed stress sensors [3]. The sensor measures the stress by detecting the polarization model coupling induced by the external transverse forces. The usage of a long PMF ensures distributed stress sensing over a long distance. Because of the GBD of the PMF, the spatial resolution of stress measurements deteriorates with fiber distance. Characterization of the GBD of the long PMF mitigates the impact of birefringence dispersion on distributed stress measurements.

White-light-interferometry-based techniques, including spectrum-domain and time-domain techniques, are widely used to characterize the GBD of a PMF [4-10]. The interferogram of optical intensity as a function of wavelength or scanning length is detected in a spectrum-domain/time-domain technique. The phase of the interferogram is retrieved, which leads to the phase birefringence, group birefringence and the GBD of the PMF. The accuracy of these techniques is determined by the accuracy of the phase retrieval. For a long PMF, the interference contrast of the interferogram is low because of the short coherent length of the employed white light source. Low interference contrast results in the large error of the retrieved phase and, hence, has an impact on the accuracy of birefringence dispersion measurement. To implement precise measurements, the maximum measurable fiber length of the white-light-interferometry-based technique is severely shortened. 
Kerr phase-interrogators have been reported for measuring the differential group delay (DGD) of two sinusoidally modulated optical signals (SMOSs) [11,12], and they have been employed to measure the GBD of a Panda PMF by directly measuring the wavelength-dependent DGD of two SMOSs polarized along the principal axes of the Panda PMF [13]. The reported approach utilizes the Kerr effect instead of the interference between two polarization modes to measure the GBD, and hence removes the limitation of laser coherence length on the maximum measurable fiber length. Unfortunately, the reported approach fails when the GBD of the PMF under test is so low that the detected optical power cannot oscillate over one sinusoidal circle as the laser wavelength of the SMOSs scans. In addition, the performance of this approach has not been rigorously discussed.

In the present study, the Kerr-phase-interrogator-based technique is systematically studied for measuring the GBD of a PMF. The GBD of a Panda PMF and an elliptical core PMF are measured by the proposed technique to demonstrate the high GBD measurement and the low GBD measurement. In addition, the performance of the proposed technique is rigorously analyzed. With the compensation for the intensity loss of the SMOSs, the proposed approach is capable of characterizing a PMF that is tens of kilometers long.

\section{Experimental Setup}

The schematic of the Kerr phase-interrogator for GBD measurements is presented in Figure 1. A continuous light-wave (CW) from a tunable laser (TL) operates at wavelength $\lambda$ and is amplitude-modulated by an electro-optic modulator (EOM). The EOM is driven by a sinusoidal electrical signal oscillating at radio frequency $f_{m}$ and turns the CW into an SMOS oscillating at a radio frequency $f_{s}=2 f_{m}$. The optical spectrum of the resulting SMOS is comprised of two distinct sidebands separated by $f_{s}$ and centered at $v_{0}$. A polarization beam splitter (PBS) splits the SMOS into two orthogonal polarization components. The two orthogonal polarization components propagate through the fast axis and slow axis over equal fiber length, respectively. A polarization beam combiner (PBC) combines the two orthogonal polarization components and sends them into a fiber under test (FUT) through a polarization-maintaining circulator (PMC). Reflected at the far end of the FUT by a polarization-maintaining reflector $\mathrm{R}$, the combined signals are amplified by an erbium-doped fiber amplifier (EFDA) and are injected into a Kerr medium comprised of a $2 \mathrm{~km}$ dispersion-shifted fiber (DSF, Fujikura) with waveguide nonlinearity of $2.28 \mathrm{~W}^{-1} / \mathrm{km}$. The amplitudes of the amplified combined signals are identical, which is realized by finely adjusting the polarization state of the SMOS with a polarization controller (PC) placed before the PBS. In the DSF, the Kerr effect results in the distinct sidebands in the total optical spectrum of the combined signals, and the power of the first-order sideband $P_{1}$ is expressed as [11,12]:

$$
P_{1}=P_{10}\left[1+\cos \left(2 \pi f_{s} t_{d}+\phi_{0}\right)\right]
$$

where $P_{10}$ is a constant value, $\phi_{0}$ is the constant phase and $t_{d}$ is the DGD representing the round-trip time differential group delay between the slow axis and fast axis of the FUT.

Using the Hilbert transform and arctan function [12], the relative phase $\Delta \phi(\lambda)=\phi(\lambda)-\phi_{\text {ref }}$ is demodulated from $P_{1}(\lambda)$, where $\phi(\lambda)=2 \pi f_{s} t_{d}(\lambda)+\phi_{0}$, and $\phi_{r e f}=2 \pi f_{s} t_{r e f}+\phi_{0}$ with $t_{\text {ref }}$ being the absolute DGD at a reference wavelength $\lambda_{\text {ref }}$. The relative DGD $\Delta t_{d}(\lambda)=t_{d}(\lambda)-t_{\text {ref }}$ is obtained by

$$
\Delta t_{d}(\lambda)=\frac{\Delta \phi(\lambda)}{2 \pi f_{s}} .
$$




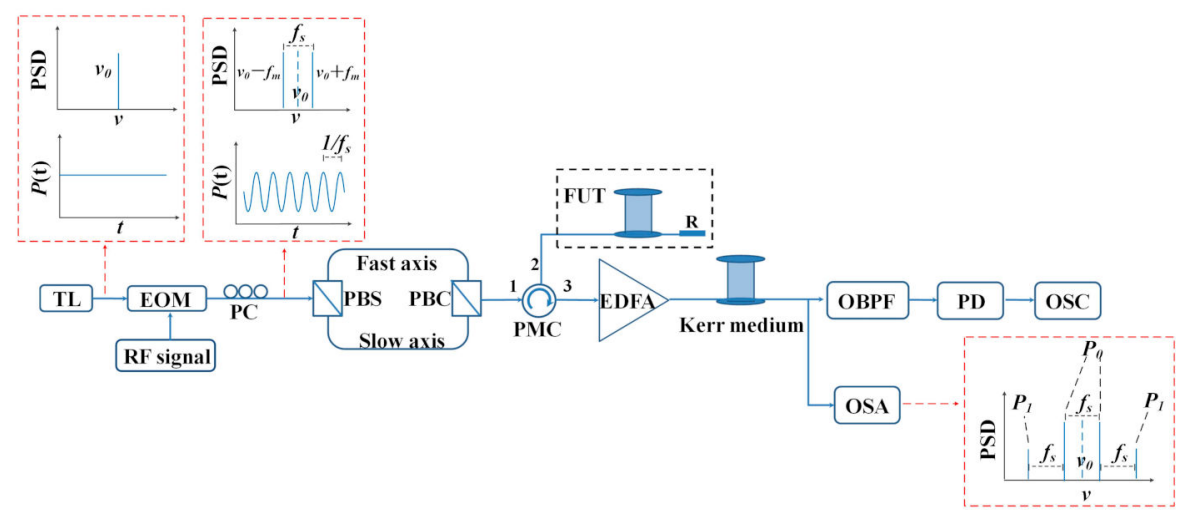

Figure 1. Schematic of the Kerr phase-interrogator for group birefringence dispersion (GBD) measurements. TL: tunable laser; EOM: electro-optic modulator; RF: radio frequency; PC: polarization controller; PBS: polarization beam splitter; PBC: polarization beam combiner; PMC: polarization-maintaining circulator; FUT: fiber under test; R: reflector; EDFA: erbium-doped fiber amplifier; OSA: optical spectrum analyzer; OBPF: optical band-pass filter; PD: photodiode; OSC: oscilloscope; PSD: power spectral density.

To measure $t_{r e f}$, the setup in Figure 1 is modified. Specifically, a $3 \mathrm{GHz}$ optical band-pass filter (OBPF), a photodetector (PD) and an oscilloscope (OSC) are cascaded to record $P_{1}$ as a function of $f_{s}$. In the modified setup, the laser wavelength of the two SMOSs $\lambda$ is set at a reference wavelength $\lambda_{\text {ref }}$ and $f_{s}$ is linearly scanned, which results in the sinusoidal variation of $P_{1}\left(f_{s}\right)$ according to Equation (1). The relative phase $\Delta \phi\left(f_{s}\right)=\phi\left(f_{s}\right)-\phi_{\text {init }}$ is demodulated from $P_{1}\left(f_{s}\right)$, where $\phi_{\text {init }}=2 \pi f_{s-\text { init }} t_{\text {ref }}+\phi_{0}$ and $f_{s-i n i t}$ is the initial value of $f_{s}$. $t_{\text {ref }}$ is calculated from the slope of $\phi\left(f_{s}\right)$ with

$$
t_{r e f}=\frac{1}{2 \pi} \frac{d}{d f_{s}}\left[\Delta \phi\left(f_{s}\right)\right]
$$

$t_{r e f}$, another absolute DGD $t_{r e f 2}$ at a wavelength $\lambda_{\text {ref } 2}$, needs to be measured and then compared with $t_{\text {ref }}$ to specify the actual sign of $\Delta t_{d}(\lambda)$. In other words, to verify whether $t_{d}(\lambda)$ increases or decreases with $\lambda$.

The absolute DGD is given by $t_{d}(\lambda)=t_{\text {ref }}+\Delta t_{d}(\lambda)$, resulting in

$$
B(\lambda)=\frac{c}{2 L}\left[t_{d}(\lambda)\right]
$$

where $B, c$ and $L$ are the group birefringence, speed of light in vacuum and the length of the FUT, respectively. Equation (4) leads to the GBD calculated by

$$
D_{B}(\lambda)=\frac{d}{d \lambda}[B(\lambda)]
$$

where $D_{B}$ represents the GBD.

\section{Experimental Results}

The GBD of a $459.4 \mathrm{~m}$ Panda PMF (Fujikura) is experimentally investigated. The wavelength of the combined signals oscillating at $f_{s}=20 \mathrm{GHz}$ is scanned from $\lambda_{1}=1540 \mathrm{~nm}$ to $\lambda_{2}=1565 \mathrm{~nm}$ in steps of $0.1 \mathrm{~nm}$, and the power spectral densities (PSDs) of the optical signal at the output of the Kerr medium are recorded for each $\lambda$ by the optical spectrum analyzer (OSA). The recorded values of $P_{1}$ as a function of $\lambda$ are presented in Figure 2a, showing a sinusoidal power variation as $\lambda$ increases, which agrees with the theoretical analysis and the indication of Equation (1). The relative phase $\Delta \phi(\lambda)$ is calculated from $P_{1}(\lambda)$ with $\lambda_{\text {ref }}=\lambda_{1}$, and is presented in Figure $2 \mathrm{~b}$. 


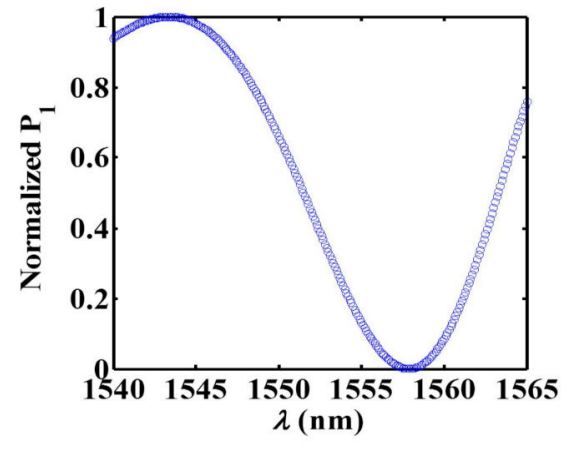

(a)

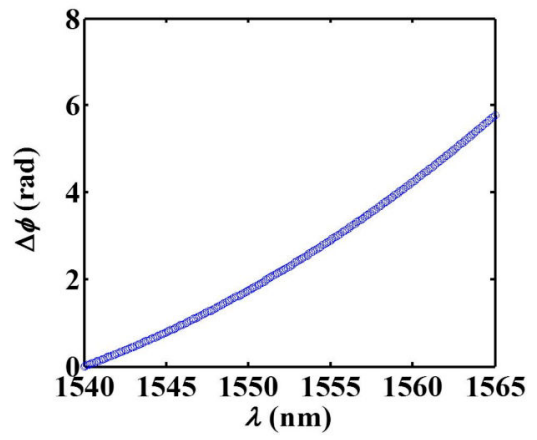

(b)

Figure 2. Measurement results of (a) $P_{1}$ and (b) $\Delta \phi$ as a function of $\lambda$ in the case of Panda polarization-maintaining fiber (PMF) characterization.

To measure the absolute DGD of $t_{r e f}$, the reference wavelength is fixed at $\lambda_{r e f}=\lambda_{1} . f_{s}$ is linearly scanned at a rate of $r=80 \mathrm{GHz} / \mathrm{s}$ from $f_{s-i n i t}=16 \mathrm{GHz}$ to $f_{s-\text { fin }}=24 \mathrm{GHz}$, and the sideband power $P_{1}$ as a function of time $t$ is acquired by an oscilloscope. To obtain $P_{1}$ as a function of $f_{s}, t$ is replaced with $f_{s}=f_{s-i n i t}+r t$. Figure 3a presents the $P_{1}\left(f_{s}\right)$ showing a sinusoidal variation of $P_{1}$ as $f_{s}$ increases, in agreement with the indication of Equation (1). $\Delta \phi\left(f_{s}\right)$ is demodulated from $P_{1}\left(f_{s}\right)$ and is presented in Figure 3b. The linear fit $\Delta \phi_{f i t}\left(f_{s}\right)$ is also presented in Figure 3b. Applying $\Delta \phi_{f i t}\left(f_{s}\right)$ to Equation (3) gives an absolute DGD of $t_{r e f}=1448 \mathrm{ps}$ at $\lambda_{\text {ref }}=\lambda_{1}$. Similarly, a value of $t_{r e f 2}=1493 \mathrm{ps}$ is obtained at $\lambda_{r e f 2}=\lambda_{2}$. Comparison of $t_{r e f}$ and $t_{r e f 2}$ reveals the increment of $t_{d}$ as $\lambda$ increases.

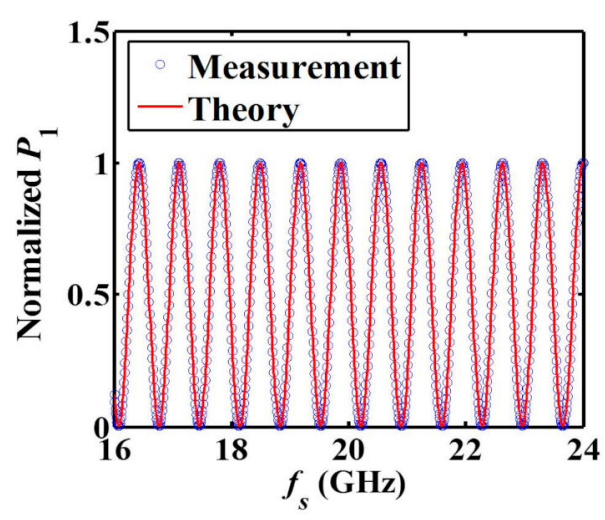

(a)

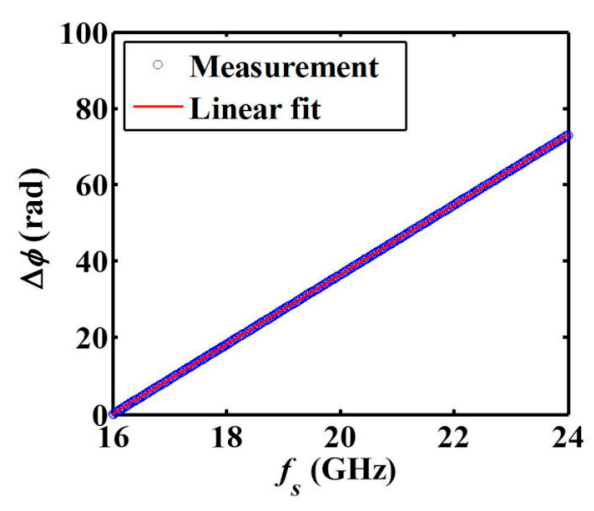

(b)

Figure 3. Measurement results of (a) $P_{1}$ and (b) $\Delta \phi$ as a function of $f_{s}$ when the laser wavelength is $\lambda_{1}$ $=1540 \mathrm{~nm}$ in the case of Panda PMF characterization.

The values of absolute DGD are calculated using $t_{d}(\lambda)=t_{r e f}+\Delta t_{d}(\lambda)$ and are presented in Figure 4a. $B(\lambda)$ is calculated from $t_{d}(\lambda)$ by using Equation (4) and $L=459.4 \mathrm{~m}$, and it is presented in Figure $4 \mathrm{~b}$ showing a value of $4.784 \times 10^{-4}$ at $\lambda=1550 \mathrm{~nm}$. Figure $4 \mathrm{c}$ presents the values of GBD, $D_{B}$, calculated from the wavelength dependence of $B(\lambda)$ by utilization of Equation (5), showing $D_{B}=5.55 \times 10^{-7} / \mathrm{nm}$ at $\lambda=1550 \mathrm{~nm}$. 


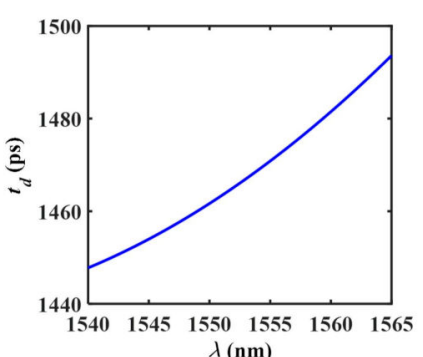

(a)

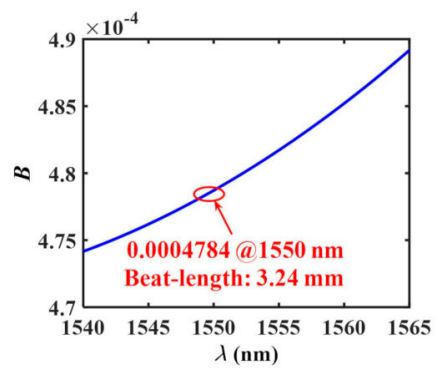

(b)

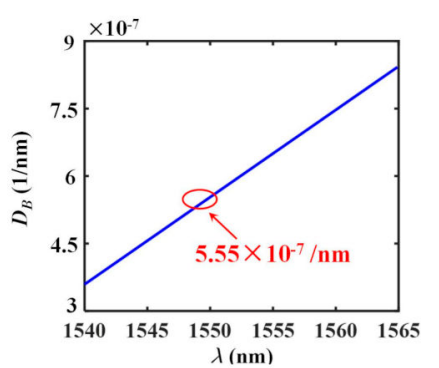

(c)

Figure 4. (a) Experimental results of (a) $t_{d}$, (b) $B$ and (c) $D_{B}$ as a function of $\lambda$ in the case of Panda PMF characterization.

Essentially, $\Delta \phi(\lambda)$ retrieval is the key for the measurements of $B(\lambda)$ and $D_{B}(\lambda)$. Obtaining $\Delta \phi(\lambda)$, one can easily calculate $B(\lambda)$ and $D_{B}(\lambda)$ directly from $\Delta \phi(\lambda)$ using Equations (2)-(5). $\Delta \phi(\lambda)$ is retrieved from the sinusoidal variation of $P_{1}(\lambda)$ [12]. Accurate phase retrieval of $\Delta \phi(\lambda)$ requires $P_{1}(\lambda)$ to undergo at least one sinusoidal variation cycle as $\lambda$ varies over the wavelength range of interest. In other words, a maximum relative phase of $\Delta \phi^{\max }$ larger than $2 \pi$ is expected. For an FUT with the low value of $\left|D_{B}\right|$ over the wavelength range of interest, the difference of $t_{d}$ among different wavelengths is small, and $\Delta \phi^{\max }$ is much smaller than $2 \pi$. In this scenario, $\Delta \phi(\lambda)$ cannot be retrieved accurately, and the GBD measurement fails. To realize measurements in this scenario, the experimental setup in Figure 1 is modified. Rather than directly measuring the $t_{d}$ of two orthogonally polarized SMOSs, the modified setup first measures the wavelength-dependent group delays of fast axis $t_{f}(\lambda)$ and slow axis $t_{s}(\lambda)$, respectively. Then, $t_{d}(\lambda)$ is calculated by $t_{d}(\lambda)=t_{s}(\lambda)-t_{f}(\lambda)$. Application of $t_{d}(\lambda)$ to Equation (4) leads to $B(\lambda)$. Accordingly, $D_{B}(\lambda)$ is obtained by utilization of Equation (5).

Figure 5 presents the modified measurement setup to interrogate $t_{f}(\lambda)$ and $t_{s}(\lambda)$. In the experiment, a 509.5 m elliptical core PMF (Draka) is under test. To measure $t_{f}(\lambda)$, port 1 and port 3 of the PMC with the FUT and the reflector $\mathrm{R}$ are connected to the fast axis ports of the PBS and the PBC, respectively. Using this setup, the difference of $t_{f}$ and $t_{s-r e f}$ is measured, where $t_{s-r e f}$ refers to the group delay of the SMOS propagating from the input of the PBS to the slow axis port of the PBC. The process of $t_{f}(\lambda)-t_{s-r e f}(\lambda)$ measurement is the same as that of $t_{d}(\lambda)=t_{s}(\lambda)-t_{f}(\lambda)$ measurement in the case of the Panda PMF. Similarly, $t_{s}(\lambda)-t_{f-r e f}(\lambda)$ is obtained by connecting port 1 and port 3 of the PMC to the slow axis ports of the PBS and the PBC, respectively, where $t_{f-r e f}$ refers to the group delay of the SMOS propagating through the fast axes of the PBS and the PBC. Then, $t_{d}(\lambda)$ is calculated by

$$
t_{d}=\left[t_{s}(\lambda)-t_{f}(\lambda)\right]-\left[t_{f-r e f}(\lambda)-t_{s-r e f}(\lambda)\right]
$$

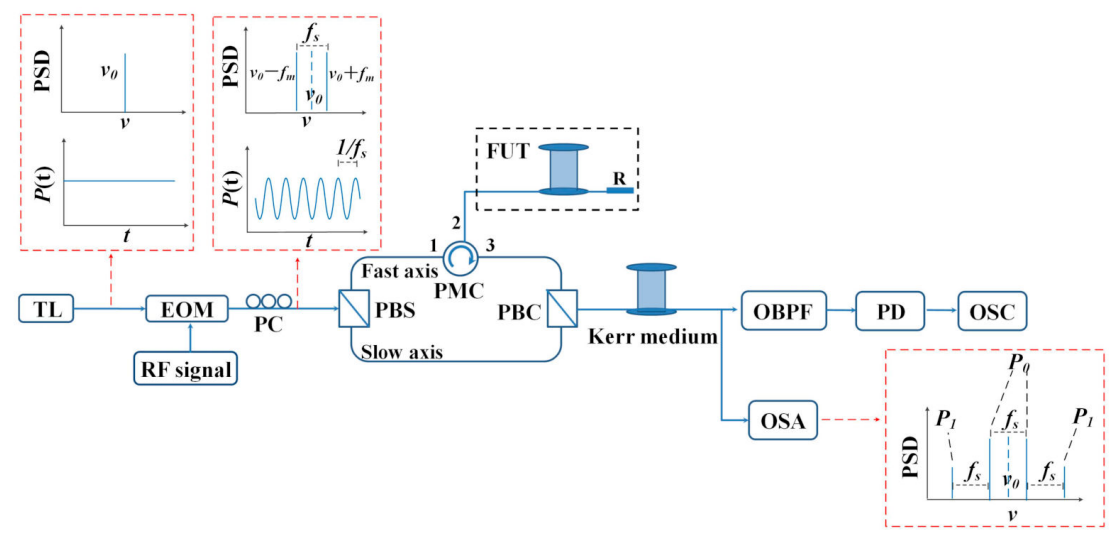

Figure 5. Schematic of the modified measurement setup to interrogate $t_{f}(\lambda)$ and $t_{s}(\lambda)$ in the case of elliptical core PMF characterization. 
Considering that the fiber length of the connection between the PBS and the PBC is much shorter than the length of the FUT, $\left|t_{f-r e f}(\lambda)-t_{s-r e f}(\lambda)\right|$ is much smaller than $\left|t_{s}(\lambda)-t_{f}(\lambda)\right|$. As a result, the approximation of $t_{d}(\lambda)=t_{s}(\lambda)-t_{f}(\lambda)$ is valid. With Equations (4) and (5), $B(\lambda)$ and $D_{B}(\lambda)$ are calculated.

Figure 6a presents the measurement results of group delays $t_{f}(\lambda), t_{s}(\lambda)$ and calculated DGD $t_{d}(\lambda)$. It is shown that $t_{f}$ and $t_{s}$ decline as $\lambda$ increases. In contrast, $t_{d}$ declines in the beginning and then increases after a turning point at $1555 \mathrm{~nm}$. Wavelength-dependent group birefringence $B(\lambda)$ of the elliptical core fiber is calculated from $t_{d}(\lambda)$ by usage of Equation (4) with $L=509.5 \mathrm{~m}$, and the values are presented in Figure $6 \mathrm{~b}$. The results show $B=4.553 \times 10^{-4}$ at $\lambda=1550 \mathrm{~nm}$. $D_{B}$ is calculated from $B(\lambda)$ by utilization of Equation (5), and the calculated values are presented in Figure 6c. The calculated results reveal that GBD is normal with $D_{B}<0$ for $\lambda<1555 \mathrm{~nm}$ and anomalous with $D_{B}>0$ for $\lambda>1555 \mathrm{~nm}$.

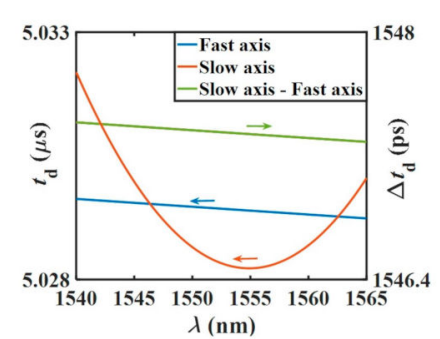

(a)

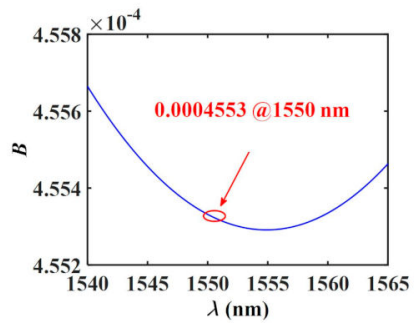

(b)

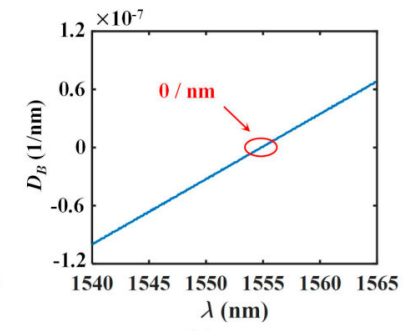

(c)

Figure 6. Experimental results of (a) $t_{f}, t_{s}$ and calculated $t_{d},(\mathbf{b})$ group birefringence $B$ and (c) group birefringence dispersion $D_{B}$ as a function of the laser wavelength $\lambda$ in the case of elliptical core PMF characterization.

\section{Discussion}

The precision of the proposed approach is determined by the minimum detectable relative phase $\Delta \phi^{\text {min }}=2 \pi f_{s} \Delta t_{d}^{\min }=\alpha$. Using $\alpha=0.02 \mathrm{rad}$ [14] and $f_{s}=20 \mathrm{GHz}$ leads to the minimum detectable relative DGD of $\Delta t_{d}^{\min }=159 \mathrm{fs}$. The precision can be further improved by usage of commercially available $50 \mathrm{GHz}$ electro-optic modulators allowing for $f_{s}=100 \mathrm{GHz}$ and $\Delta t_{d}^{\min }=32 \mathrm{fs}$. In the case of elliptical core PMF characterization, $\Delta t_{d}^{\min }$ is doubled because relative DGD in this case is obtained by the measurement of group delays of two principal axes, rather than by one direct measurement of DGD. Two measurements make $\Delta t_{d}^{\min }$ twofold.

For the measurement of GBD, the limits of the proposed approach are estimated from the DGD variation rate $\rho=D_{B} \times 2 L / c$ [12]. Applications of Equation (4), Equation (5) and $t_{d}(\lambda)=t_{r e f}+\Delta t_{d}(\lambda)$ lead to $\rho=\delta t_{d} / \delta \lambda$, where $\delta t_{d}$ and $\delta \lambda$ respectively are the DGD difference and wavelength separation between two adjacent scanning wavelengths. In the case of Panda PMF characterization, the minimum measurable DGD variation rate is $\rho^{\min }=\delta t_{d}^{\min } / \delta \lambda^{\max }$ with $\delta \lambda^{\max }$ being the maximum laser scanning range. In our experimental setup, the EDFA works in C band, and thus $\delta \lambda^{\max }$ is limited by $30 \mathrm{~nm}$. Applications of $\delta t_{d}^{\min }=\Delta t_{d}^{\min }=159 \mathrm{fs}$ and $\delta \lambda^{\max }=30 \mathrm{~nm}$ lead to $\rho^{\min }=5.3 \mathrm{fs} / \mathrm{nm}$. The maximum measurable DGD variation rate is $\rho^{\max }=\delta t_{d}^{\max } / \delta \lambda^{\min }$ with $\delta \lambda^{\min }$ being the minimum laser scanning step. Accurate restoration of the sinusoidal power variation of $P_{1}(\lambda)$ requires $N$ sampling points within a sinusoidal variation cycle. This requirement restricts $\delta \phi^{\max }=2 \pi / N$, where $\delta \phi^{\max }=2 \pi f_{s} \delta t_{d}^{\max }$ is the maximum phase difference associated with two adjacent scanning wavelengths. Accordingly, $\delta t_{d}^{\max }=1 / N f_{s}$ and $\rho^{\max }=1 / N f_{s} \delta \lambda^{\min }$ are obtained. Applications of $N=10, f_{s}=20 \mathrm{GHz}$ and $\delta \lambda^{\min }=0.001 \mathrm{~nm}$ of our tunable laser lead to $\rho^{\max }=5 \mathrm{~ns} / \mathrm{nm}$. In the case of elliptical core PMF characterization, $\rho^{\max }$ is identical to that of the Panda PMF measurement, while $\rho^{\min }$ is double because the minimum detectable relative DGD $\Delta t_{d}^{m i n}$ is twice that in the case of Panda PMF characterization.

The maximum measurable fiber length $L^{\max }$ is determined by the maximum measurable DGD variation rate $\rho^{\max }=\delta t_{d}^{\max } / \delta \lambda^{\min }=D_{B} \times 2 L^{\max } / c$ and the intensity loss of the SMOSs traveling through the FUT. Applications of $\rho^{\max }=5 \mathrm{~ns} / \mathrm{nm}$ and $D_{B}=5.55 \times 10^{-7} / \mathrm{nm}$ of the Panda PMF at 
$\lambda=1550 \mathrm{~nm}$ lead to $L^{\max }=c \rho^{\max } / 2 D_{B} \approx 1.35 \times 10^{6} \mathrm{~m}$. Taking into account the intensity loss of SMOSs that travel through the FUT, one can place an EDFA after port 3 of the PMC in Figure 4 or Figure 5 to compensate the intensity loss and thus to extend the maximum measurable fiber length. Considering the loss of $0.2 \mathrm{~dB} / \mathrm{km}$, application of the EDFA offering $10 \mathrm{~dB}$ gain allows for a $50 \mathrm{~km}$ measurable fiber length.

The measurement sensitivity of the proposed approach in comparison with that of the white-light-interferometry-based technique is also discussed. Essentially, the proposed approach interrogates the GBD of a PMF by measuring the DGD of two SMOSs. Therefore, the measurement sensitivity is governed by that of the DGD measurement. As Equation (1) reveals, the DGD is demodulated from the sinusoidal power variation $P_{1}$ that oscillates at a frequency $f_{s}$ on the order of $10^{10} \mathrm{~Hz}$. Consequently, the sensitivity of DGD measurement is determined by $f_{s}$. Similarly, the white-light-interferometry-based technique interrogates the GBD of a PMF by measuring the phase delay of two optical signals. The phase delay is demodulated from the sinusoidal power variation that oscillates at an optical frequency $v$, and the measurement sensitivity of the white-light-interferometry-based technique is determined by $v$. For a light wave operating at $C$ band, the optical frequency is on the order of $10^{14} \mathrm{~Hz}$, which is $10^{4}$ higher than $f_{s}$. Therefore, the measurement sensitivity of the white-light-interferometry-based technique is $10^{4}$ higher than that of the proposed approach.

\section{Conclusions}

In conclusion, the Kerr-phase-interrogator-based method is systematically studied to measure the GBD of a long PMF, and two commercial PMFs with high GBD and low GBD are experimentally investigated. Rather than utilizing the interference between two polarization modes, the proposed approach employs the Kerr effect between two polarization modes to interrogate the GBD of a PMF. Therefore, the proposed approach is free from the limitation of laser coherent length on the measurable fiber length. By using a commercial EDFA, the intensity loss of the SMOSs can be compensated, which allows for characterizing a PMF with a length of tens of kilometers.

Funding: This work was supported by the Natural National Science Foundation of China (NSFC) (61705262, 61775238, 61705263), and the National Basic Research Program of China (2017YFB0405503).

Conflicts of Interest: The author declares no conflict of interest.

\section{References}

1. Jinno, M.; Matsumoto, T. Ultrafast, low power, and highly stable all-optical switching in an all polarization maintaining fiber Sagnac interferometer. IEEE Photon. Technol. Lett. 1990, 2, 349-351. [CrossRef]

2. Nishizawa, N.; Goto, T. Widely wavelength-tunable ultrashort pulse generation using polarization maintaining optical fibers. IEEE J. Sel. Top. Quant. Electron. 2001, 7, 518-524. [CrossRef]

3. Dixon, A.R.Z.; Yuan, L.; Dynes, J.F.; Sharpe, A.W.; Shields, A.J. Gigahertz decoy quantum key distribution with 1Mbit/s secure key rate. Opt. Express 2008, 16, 18790-18797. [CrossRef] [PubMed]

4. Flavin, D.A. Dispersion of birefrinigence and differential group delay in polarization-maintaining fiber. Opt. Lett. 2002, 27, 1010-1012.

5. Hlubina, P. Dispersion of group and phase modal birefringence in elliptical-core fiber measured by white-light spectral interferometry. Opt. Express 2003, 11, 2793-2798. [PubMed]

6. Chen, X.; Zhang, H.; Jia, D.; Liu, T.; Zhang, Y. Spectral-domain measurement of chromatic dispersion difference of polarization modes in polarization-maintaining fibers. J. Mod. Optic. 2011, 58, 26-31. [CrossRef]

7. Deng, D.; Sega, D.; Cheng, T.; Gao, W.; Xue, X.; Suzuki, T.; Ohishi, Y. Dispersion characterization of two orthogonal modes in a birefringence tellurite microstructured optical fiber. Opt. Express 2014, 22, 23920-23927. [CrossRef] [PubMed]

8. Lee, J.Y.; Kim, D.Y. Versatile chromatic dispersion measurement of a single mode fiber using spectral white light interferometry. Opt. Express 2006, 14, 11608-11615. [CrossRef] [PubMed] 
9. Tang, F.; Wang, X.Z.; Zhang, Y.; Jing, W. Characterization of birefringence dispersion in polarization maintaining fibers by use of white-light interferometry. Appl. Opt. 2007, 46, 4073-4080. [CrossRef] [PubMed]

10. Yu, Z.J.; Yang, J.; Yuan, Y.G.; Li, C.; Liang, S.; Hou, L.; Peng, F.; Wu, B.; Zhang, J.Z.; Liu, Z.H.; et al. Quasi-distributed birefringence dispersion measurement for polarization maintain device with high accuracy based on white light interferometry. Opt. Express 2016, 24, 1588-1597. [CrossRef] [PubMed]

11. Baker, C.; Bao, X. Displacement sensor based on Kerr induced phase-modulation of orthogonally polarized sinusoidal optical signals. Opt. Express 2014, 22, 9095-9100. [CrossRef] [PubMed]

12. Baker, C.; Lu, Y.; Bao, X. Chromatic-dispersion measurement by modulation phase-shift method using a Kerr phase-interrogator. Opt. Express 2014, 22, 22314-22319. [CrossRef] [PubMed]

13. Lu, Y.; Baker, C.; Bao, X. Dispersion Characterization of Group Birefringence in Polarization-Maintaining Fiber using a Kerr Phase-Interrogator. In Proceedings of the 5th Asia Pacific Optical Sensors Conference (APOS 2015), Jeju, Korea, 20-22 May 2015.

14. Lu, Y.; Baker, C.; Chen, L.; Bao, X. Chromatic-dispersion monitor based on a differential phase-shift method using a Kerr phase-interrogator. IEEE Photonics J. 2015, 7, 7101406. [CrossRef]

Publisher's Note: MDPI stays neutral with regard to jurisdictional claims in published maps and institutional affiliations.

(C) 2020 by the author. Licensee MDPI, Basel, Switzerland. This article is an open access article distributed under the terms and conditions of the Creative Commons Attribution (CC BY) license (http://creativecommons.org/licenses/by/4.0/). 